\title{
Quando la paura guida le scelte. Donne immigrate e salute riproduttiva*
}

\author{
di Francesco Della Puppa**, Pamela Pasian ${ }^{* * *}$ \\ e Giuliana Sanò
}

Riassunto: L'articolo discute i risultati di una ricerca etnografica condotta nel Comune di Verona, nel corso del 2018, e finalizzata all'analisi dei bisogni delle donne migranti relativamente alle fasi di accesso e presa in carico da parte dei servizi territoriali dedicati alla salute riproduttiva. La ricerca ha messo in luce che, oltre a una serie di criticità di natura strutturale-organizzativa, a disincentivare il ricorso ai servizi contribuisce un ostacolo di natura squisitamente emotiva e, nello specifico, il sentimento della paura. In questo contributo, quindi, si proverà a riflettere sul ruolo esercitato dalla paura nella relazione tra donne immigrate e servizi dedicati alla salute riproduttiva, interpretando tale sentimento non già come l'espressione di un vissuto e di un sentito meramente individuale, piuttosto come "un pensiero incorporato", esito di una costruzione sociale, riflesso di dinamiche e relazioni di potere, capaci di trasformare i sentimenti in pratiche.

Parole-chiave: donne immigrate; salute riproduttiva; emozioni; paura; servizi; tattiche.

\section{When fear drives the choices. Migrant women and reproductive}

Abstract: The article discusses the results of an ethnographic research conducted in the Municipality of Verona, during 2018, and aimed at analysing the needs of migrant women due to the access and taking charge by the territorial services for their reproductive health. The research has highlighted that, in addition to many structural-organizational critical issues, a merely emotional obstacle contributes to discouraging the use of services, that is, specifically, the feeling of fear. In this paper,

* Questo articolo è frutto di un lavoro collettivo, congiunto e indivisibile. Tuttavia, se devono essere assegnate delle attribuzioni individuali, Francesco Della Puppa ha scritto l'introduzione e i paragrafi 1; 2; 3; Pamela Pasian ha scritto i paragrafi 4.2; 4.4; Giuliana Sanò ha scritto i paragrafi 4; 4.1; 4.3 e le Conclusioni.

**. Francesco Della Puppa, Università Ca' Foscari Venezia (Italia), Dipartimento di Filosofia e Beni Culturali; francesco.dellapuppa@unive.it.

***. Pamela Pasian, Università Ca' Foscari Venezia (Italia), Dipartimento di Filosofia e Beni Culturali; pamela.pasian@unive.it.

****. Giuliana Sanò, Università degli Studi di Messina (Italia), Dipartimento di Scienze Cognitive, Psicologiche, Pedagogiche e degli Studi Culturali; giulianasano@hotmail.it.

DOI: 10.3280/MM2020-003005

Mondi Migranti, (1972-4888, ISSNe 1972-4896), 3/2020 
therefore, we will try to reflect on the role exercised by fear in the relationship between migrant women and services for reproductive health. We interpret this emotion not as the expression of a merely individual experience and feeling, rather than as "an embedded thinking", the result of a social construction that reflects dynamics and power relationships, capable of transforming feelings into practices.

Keywords: migrant women; reproductive health; emotions; fear; services; tactics.

$$
\text { Ricevuto: } 01 \text { Marzo } 2020
$$

Accettato: 07 Maggio 2020

\section{Introduzione}

Gli approfondimenti sulla relazione tra servizi socio-sanitari e utenza immigrata si sono interrogati frequentemente sugli impedimenti e sulle ragioni che possono inibirne l'accesso e, di conseguenza, il godimento di prestazioni mediche, sociali e sanitarie; sulle ricadute per la salute pubblica di tali ostacoli e sulle eventuali misure da adottare per superarli (Quaranta e Ricca, 2012; Terraneo e Tognetti Bordogna, 2018; Tognetti Bordogna, 2012). Fra i fattori che possono rendere difficoltoso l'accesso di utenti immigrati alla tutela della salute da parte delle strutture pubbliche, un ruolo spesso determinante lo svolgono specifiche emozioni e stati d'animo, indotti da specifiche condizioni materiali, sociali e personali.

Nelle prossime pagine, quindi, verranno presentati i risultati di una ricerca etnografica finalizzata all'analisi dei bisogni delle donne migranti relativamente alle fasi di accesso e presa in carico da parte dei servizi territoriali dedicati alla salute riproduttiva, che ha messo in luce come, oltre a una serie di criticità di natura strutturale-organizzativa, a disincentivare il ricorso ai servizi contribuisce un ostacolo di natura squisitamente emotiva, appunto, e, nello specifico, il sentimento della paura. Si proverà, cioè, a riflettere sul ruolo esercitato dalla paura nella relazione tra donne immigrate e servizi dedicati alla salute riproduttiva, interpretando tale sentimento non già come l'espressione di un vissuto e di un sentito meramente individuale, ma come "un pensiero incorporato", esito di una costruzione sociale, riflesso di dinamiche e relazioni di potere, capaci di trasformare $i$ sentimenti in pratiche.

\section{Per un'etnografia delle emozioni}

Le emozioni possono essere concettualizzate come un modo di essere e di stare nel mondo sociale, un mezzo per interpretarlo, categorizzarlo, percepirlo e, di conseguenza, agire in esso (Pussetti, 2005). Esse costituirebbero, cioè, un "pensiero incorporato" (Rosaldo, 1984) che precede, determina 
e orienta l'azione (Bourdieu, 1972).

È possibile, infatti, interpretare le emozioni come "modelli di esperienza acquisiti" (Pussetti, 2005), forgiati dall' apprendimento di determinati ordini socio-culturali e normativi e storicamente situati. In altri termini, le emozioni vanno intese sia come costruzioni sociali e culturali (Geertz, 1973), sia come riflessi sovrastrutturali della struttura sociale che si esprimono a livello corporeo, sulla base della biografia personale dei singoli individui, e che interessano un "Io corporeo" (Rosaldo, 1984), posto all'intersezione tra individuo, corpo e società (McCarthy, 2017; Thoits, 1996; Pussetti, 2005). Le emozioni, cioè, da un lato, sono frutto di uno specifico "discorso egemonico" e di uno specifico sistema di relazioni sociali, dall'altro lato esse li legittimano e li riproducono (Abu-Lughod and Lutz, 1990; Lutz, 1988).

Ciò, ovviamente, non implica che le emozioni, parimenti alla violenza simbolica (Bourdieu, 1998) di cui le emozioni si imbevono (Toffanin, 2015), siano rigidamente determinate, che neghino qualunque possibilità di agency individuale, di resistenza soggettiva, di libertà emozionali (Reddy, 1999) o lo scostamento da una riproduzione socio-culturale lineare e, con esse, la possibilità del cambiamento storico. È necessario, infatti, prendere in considerazione la complessità esperienziale degli individui, i mutamenti storici delle relazioni sociali e strutturali e la loro incidenza sul contesto emozionale (Bordonaro, 2005). Al contempo, inoltre, le emozioni possono essere soggette al "lavoro" degli stessi individui che le provano e le incorporano e vengono da esso modellate (Bolton, 2005; Hochschild, 1979).

Accanto a un approccio che possiamo definite "discorsivo" (Abu-Lughod and Lutz, 1990; Lutz, 1988; Pussetti, 2005) e "costruzionista" (Harré, 1986), è possibile rintracciare un approccio interazionista (Cerulo, 2019; Sandstrom et al., 2013), secondo il quale le emozioni costituiscono un duplice strumento ermeneutico, necessario, da un lato, a studiare la dimensione collettiva e individuale della realtà sociale e, dall'altro, a innescare un processo di consapevolezza riflessiva e personale (van Zomeren, 2016; Fields et al., 2007). Secondo un simile approccio, cioè, si creerebbe un processo circolare tra emozioni e consapevolezza: attraverso le espressioni emotive, gli individui riescono a stare e agire nei diversi contesti situazionali della vita quotidiana; mentre attraverso il controllo di tali espressioni, coerentemente con i contesti situazionali nei quali sono collocati, essi possono interagire con altri individui e innescare nuovi meccanismi di riflessività per comprendere se stessi (Ibidem). Da questo punto di vista, quindi, le emozioni sono prodotte e riprodotte, definite e ridefinite, acquistando senso e significati, a seconda dell'interazione in cui vengono "scambiate" e "comunicate".

Nonostante una lunga tradizione di studi socio-antropologici sulle emozioni (Cerulo, 2018; Harris, 2015; Hochschild, 1979; Lutz and White, 1986; 
Pussetti, 2005; Rosaldo, 1984; Turner and Stets, 2005), solo pochi autori hanno, però, per utilizzato tali prospettive per leggere le dinamiche migratorie che attraversano la società (Carling, 2008; Lindquist, 2009; Baldassar, 2010; Brooks and Simpson, 2013).

Nei migration studies, la dimensione emozionale è stata presa in considerazione, soprattutto, per analizzare le spinte soggettive alla base della scelta della partenza e alle traiettorie migratorie (Ibidem) e, più frequentemente, nei contributi relativi alle relazioni familiari transnazionali (Ambrosini, 2014; Bustamante and Alemàn, 2007; Charsley and Shaw, 2006; Skrbiš, 2008) e alla famiglia, separata e/o ricongiunta, nella migrazione (Ambrosini, 2014; Baldassar, 2010; Bustamante and Alemàn, 2007; Della Puppa, 2018; Parreñas, 2008). È il caso, per esempio, dello studio di Charsley (2005) sull'infelicità dei mariti pakistani, giunti in Europa a seguito del ricongiungimento familiare con le loro mogli, appartenenti alle seconde e terze generazioni, nate e cresciute nel Regno unito; o dei diversi approfondimenti del senso di colpa provato dalle donne emigrate per la lontananza e la separazione fisica dai loro genitori anziani o dai loro figli rimasti nel Paese di origine (Baldassar, 2010; Brooks and Simpson, 2013) e delle conseguenti strategie messe in atto per fare fronte alla distanza (Bryceson and Vuorela, 2002); o, ancora, dell' ambivalenza dello scarto di genere che caratterizza le logiche emozionali di uomini e donne bangladesi, modellando le scelte e le traiettorie familiari e migratorie (Della Puppa, 2018).

Da un punto di vista analitico, quindi, le emozioni costituiscono un costrutto sociale, incorporato nelle relazioni socio-culturali (Harré, 1986) che può influenzare le scelte di mobilità e la gestione delle relazioni transnazionali degli emigrati (Baldassar, 2010).

In questa sede, invece, analizzeremo come le emozioni orientino le scelte quotidiane (Turnaturi, 2012) degli immigrati nel Paese di destinazione. Nello specifico, come anticipato, ci concentreremo su come il sentimento della paura - in quanto pensiero incorporato, appunto - condizioni le relazioni tra donne immigrate e servizi per la salute riproduttiva femminile e, spesso, ne inibisca l'accesso (Quaranta e Ricca, 2012; Terraneo e Tognetti Bordogna, 2018; Tognetti Bordogna, 2012); ci interrogheremo su cosa generi tale emozione e su quali siano le declinazioni lungo le quali essa si articola e prende forma rispetto alle modalità di accesso e fruizione dei servizi.

Le ricerche che si sono occupate di esaminare la qualità del rapporto che le donne immigrate intrattengono con i servizi sanitari hanno evidenziato la presenza di diverse difficoltà di accesso per l'utenza straniera (Ibidem). La prima difficoltà è riconducibile allo status giuridico delle donne che, in qualche caso, può disincentivare il ricorso ai servizi da parte di queste. Malgrado le criticità del pacchetto sicurezza 2009 non si siano, di fatto, concretizzate (Ferrero, 2010), è abbastanza diffusa tre le persone irregolari l'idea di correre 
un rischio qualora si dovesse presentare la necessità di ricorrere alle cure mediche o ai nosocomi.

In maniera analoga, altri fattori possono disincentivare il ricorso alle strutture sanitarie o renderne particolarmente difficile l'accesso per le donne immigrate in Italia (Censis, 2015; Lombardi, 2005; Mangone e Masullo, 2013; Quaranta e Ricca, 2012; Rossi e Tognetti Bordogna, 2017; Terraneo e Tognetti Bordogna, 2018; Tognetti Bordogna, 2012), tra questi: il titolo di studio, le competenze linguistiche, la solitudine, il tempo di permanenza sul territorio delle utenti, un apparato di conoscenze e di saperi differenti e culturalmente orientati, la burocratizzazione delle procedure mediche, la parcellizzazione dei servizi, la mancanza di informazioni o la tendenza degli operatori a dare informazioni frammentarie e, come cercheremo di mostrare di seguito, i sentimenti di paura e disorientamento sviluppati dalle utenti e frutto dell'intreccio tra condizioni strutturali e individuali.

$\mathrm{Al}$ di là della complessità comunicativa della malattia, della cura e dei corpi, esistono criticità che vanno tuttavia poste e indagate a partire dalla valenza strutturale di certi dispositivi istituzionali e sanitari. Nel rapporto tra le donne e i servizi dedicati alla salute riproduttiva si affacciano una serie di problematiche ascrivibili, in primo luogo, alla riduzione del Sé e della dimensione soggettiva nelle scelte inerenti alla sfera riproduttiva.

Tra gli aspetti che in pochi hanno messo a fuoco vi è senz'altro quello relativo alla co-costruzione dei saperi e, in particolare, di quelli legati al corpo e alla maternità (Giuffrè, 2018; Marabello e Parisi, 2018; Quagliarello, 2018). A tal proposito, possiamo rifarci a quanto sostiene Bonfanti $(2012,7)$ e cioè che "le madri non solo ricevono una formazione prenatale, ma al contempo producono un certo sapere sulla maternità" (vedi anche Ketler, 2000). Produrre "un certo sapere sulla maternità" può, dunque, voler dire che le donne facciano ricorso a più sistemi, anche contemporaneamente (Quagliarello, 2018). A riprova di ciò, va detto che la maggior parte dei fraintendimenti comunicativi e terapeutici non nascono solo dalle incomprensioni linguistiche, ma soprattutto dalla mancanza di equilibrio tra le categorizzazioni e le strategie di intervento messe in atto dai servizi e l'estrema eterogeneità degli scenari migratori (Tarabusi, 2014). Salute e migrazioni internazionali, infatti, sono da ritenersi fenomeni ed esperienze che spaziano dal personale al sociale, dal soggettivo all'oggettivo, in un'ottica processuale (Bonfanti, 2012). In tal senso, quindi, l'utenza - in questo caso, femminile - immigrata non tende "solo" a riconfigurare il sistema sanitario delle società di arrivo (Pasini e Pullini, 2002), ma anche a plasmare tutti i settori della vita sociale (Cattaneo e Dal Verme, 2005; Tognetti, 2008).

Successivamente a questa introduzione di ricostruzione del quadro bibliografico e della letteratura scientifica, seguirà una breve descrizione sulle 
scelte metodologiche attuate e, di seguito, una presentazione del contesto politico, sociale e territoriale entro il quale ha preso forma la ricerca da cui abbiamo tratto il presente contributo.

Quindi, ci focalizzeremo sulle articolazioni della paura come prodotto della combinazione tra condizioni strutturali e condizioni individuali e come motore produttore di pratiche e tattiche - nel senso attribuitogli da De Certeau (2010) - del ruolo che tale emozione svolge nell'orientare le scelte dell'utenza immigrata relativamente alla salute riproduttiva e, soprattutto, dell'influenza che essa esercita sul loro accesso ai servizi preposti, presentando rispettivamente la voce delle coordinatrici e delle operatrici degli stessi servizi e quella delle donne immigrate.

\section{Nota metodologica ed epistemologica}

Il presente contributo è frutto di una più ampia ricerca volta a indagare $\mathrm{i}$ bisogni delle donne immigrate, con o senza figli, relativamente alle tematiche della salute riproduttiva e dei servizi ad essa preposti, nel contesto socioterritoriale del Comune di Verona.

A sua volta, la ricerca costituiva un'azione del progetto Mari (Multicultural Actions Regional Immigration), finalizzato alla "Promozione dell'accesso ai servizi per l'integrazione" e finanziato dai Fondi Asilo, Migrazione Integrazione. Il progetto è stato affidato, dall'ente strumentale della Regione Veneto, "Veneto Lavoro", all'Associazione Le Fate Onlus di Verona che ha commissionato l'attività di ricerca.

La ricerca ha approfondito, da un lato, i bisogni delle donne immigrate, nelle fasi di accesso ai servizi, presa in carico e cura; dall'altro lato, le pratiche e le strategie adottate dal personale che lavora nel settore della salute riproduttiva.

Per quanto concerne il primo focus, occorre segnalare che sono stati analizzati e presi in esame i bisogni di donne appartenenti a quattro specifiche categorie: richiedenti protezione internazionale, lungosoggiornanti, "irregolari" (Stp, Straniero/a Temporaneamente Presente) e vittime di tratta. La scelta di distinguere le donne intervistate sulla scorta della condizione giuridica non ha, qui, la funzione di assecondare il piano di distinzioni e categorizzazioni prodotte in ambito istituzionale, ma giova a evidenziare i punti di contatto tra le diverse categorie, le contraddizioni che le attraversano e, soprattutto, a storicizzare la cornice normativa e politica entro cui queste donne si muovono e vivono. Nel corso dell' attività empirica, infatti, è emerso che tali profili, inizialmente delineati, si sfumano vicendevolmente e si sovrappongono l'un l'altro a seconda del percorso biografico e migratorio delle donne 
protagoniste della ricerca e della prospettiva di osservazione considerata.

Occuparsi di salute riproduttiva significa, ovviamente, comprendere una molteplicità di tematiche, specifiche ed eterogenee, che meriterebbero di essere approfondite separatamente. In questa sede, però, non vogliamo indagare la specificità di ognuno dei singoli temi, quanto focalizzarci sul sentimento di paura che li attraversa e li accomuna, nella consapevolezza che tale sentimento, in ogni donna, si declina in maniera differente e non produce le medesime conseguenze, per ogni specifico aspetto e vissuto relativo alla sfera della salute riproduttiva.

Analogamente, abbiamo scelto di non prendere in considerazione l'origine nazionale e linguistico-culturale come elemento fondativo della nostra analisi. Se è vero, infatti, che le emozioni costituiscono un prodotto sociale e, quindi, ogni individuo è attraversato e animato da emozioni, socialmente costruite in maniera diversa, a seconda della diversa nazionalità di origine $\mathrm{e}$ del differente contesto socio-culturale in cui è stato socializzato, è altresì vero che, all'interno del medesimo contesto nazionale, sociale e culturale, vanno presi in considerazione l'intersezione dei diversi elementi costitutivi l'identità sociale dei soggetti (collocazione di classe, caratteristiche somatiche, orientamento sessuale, identità di genere, appartenenza religiosa, età anagrafica, etc.) e tutti gli altri elementi che compartecipano a modellare gli habitus degli individui, ivi compresi le esperienze personali, i capitali a disposizione, la mobilità migratoria, la socializzazione familiare... La nostra scelta, quindi, è dettata, da un lato, dall'impossibilità di poter tenere assieme tale complessità nello stesso focus analitico e, dall'altro lato, dalla volontà di evitare interpretazioni riduzioniste di carattere culturalista ed etnicizzante.

Dopo aver analizzato la letteratura grigia esistente e aver avanzato una disamina dei dati quantitativi relativi alla presenze immigrate nel Comune di Verona, con particolare attenzione alla componente femminile, abbiamo generato e raccolto 10 interviste in profondità e quattro focus group con coordinatrici e operatrici dei servizi (consultori, ambulatori per immigrati "irregolari", Centri di accoglienza, ospedali); 10 interviste in profondità e 3 focus group con donne immigrate utenti dei servizi (concentrandoci con donne Stp, richiedenti asilo, vittime di tratta, lungosoggiornanti, nonostante i limiti, già descritti, che tale categorizzazione ha mostrato).

\section{Una premessa necessaria. Diritti diseguali e tutele negate}

Prima di prendere in considerazione le narrazioni del personale e dell'utenza immigrata dei servizi per la salute riproduttiva, riteniamo neces- 
sario fare una premessa relativamente al quadro normativo, alla sua applicazione nel territorio considerato, alle conseguenze che ciò comporta sullo stato di salute delle donne immigrate e sulle loro "traiettorie riproduttive" sulla base della loro posizione amministrativa.

Va sottolineato, infatti, che le donne - e le famiglie - immigrate prive di un regolare documento di soggiorno, le cosidette "Stp", non hanno diritto a una serie di tutele in ambito sanitario. Se i ricoveri erogati a favore degli aventi diritto sono remunerati attraverso apposito fondo del Ministero, le spese connesse alle prestazioni ambulatoriali, ai farmaci e alle altre prestazioni restano a carico delle Regioni. Tale allocazione delle spese in capo alle Regioni, nello specifico caso della Regione Veneto, costituisce un nodo cruciale alla compromissione del diritto a una serie di tutele in ambito sanitario.

Fra le tutele negate, va richiamato l'accesso allo Screening per prevenire il cancro al collo dell'utero, previsto ogni 3 anni per le donne di età compresa tra i 25 e i 64 anni. Coerentemente al quadro normativo nazionale ${ }^{1}$, per un certo periodo di tempo questa fascia della popolazione femminile ha potuto beneficiare del Pap Test gratuitamente, al pari delle donne con cittadinanza italiana. Successivamente l'usss locale, su indicazione della Regione, ha negato il Pap Test gratuito per le donne Stp, chiedendo a loro il pagamento della prestazione. Ciò, presumibilmente, dissuaderà, di qui in avanti, molte utenti dal ricorrere alla prestazione e innalzerà il carico di malattia legato al tumore della cervice uterina tra le Stp, incrementando un tasso che è già più elevato per le donne immigrate rispetto alle autoctone (Crocetti et al., 2010; Spadea et al., 2010).

Se questo costituisce il quadro nazionale e la sua articolazione regionale, vanno prese in considerazione anche alcune specificità del contesto veronese. Innanzitutto, è necessario sottolineare che l'amministrazione cittadina è guidata dal partito politico della Lega Nord che, a livello nazionale ha inasprito le già forti prese di posizione nei confronti dei lavoratori e delle famiglie immigrate, attuando politiche e proponendo decreti finalizzati a una intensa restrizione dei diritti sociali di questa categoria di soggetti - anche se tale atteggiamento è in continuità con i provvedimenti legislativi, sempre più ostili alle popolazioni immigrate, che hanno caratterizzato gli ultimi decenni di storia politica italiana, trasversalmente al colore dei governi che si sono succeduti (Basso, 2010; Della Puppa et al., 2020; Gargiulo, 2018). Nello specifico, si fa riferimento alla Legge 132 del 2018 (Della Puppa et al., 2020) che ha previsto l'abolizione della norma che regolava la protezione umanitaria e, quindi, lo status di "Protezione umanitaria" che, va ricordato, veniva concesso in una varia gamma di situazioni comprensibilmente collegate, fra

1. D.Lgs. 124/ 1998; D.Lgs. 286/1998; Dpr. 394/1999. 
le altre cose, allo stato di salute, alla maternità, alle traversie affrontate nel viaggio verso l'Italia, alla permanenza prolungata in Libia - contesto in cui gli emigrati e le emigrate sono sistematicamente sottoposti a torture, violenze, stupri. La sua abolizione, ovviamente, ha comportato e sta comportando immediate conseguenze che, per legge, non trovano ricomposizione nemmeno nel permesso per cure mediche.

Insieme all'abrogazione, di fatto, del sistema Sprar per i richiedenti asilo e titolari di protezione, il decreto ha previsto l'eliminazione del diritto all'iscrizione anagrafica degli stranieri richiedenti asilo, con la conseguenza che molte di queste persone non possono quindi avere accesso ai servizi presenti nei territori.

A livello locale, invece, la giunta comunale ha intrapreso un percorso finalizzato ad attaccare i diritti sociali e le condizioni della salute riproduttiva delle donne tout court, approvando una delibera che dichiara Verona "Città a favore della vita", ma che, di fatto, mira ad attaccare - per ora, simbolicamente - il diritto all'interruzione di gravidanza e sostiene associazioni cattoliche che mettono in campo iniziative contro l'Ivg. Tale delibera, ovviamente, ha contribuito alla creazione di un clima sociale ostile alle donne soprattutto immigrate - e ai servizi di tutela della loro salute riproduttiva.

Il Veneto, inoltre, risulta la seconda regione dell'Italia settentrionale, dopo il Trentino Alto-Adige, per crescita di medici obiettori. Nello specifico, nel contesto veronese, su 66 medici in servizio nelle unità operative di ostetricia e ginecologia, 54 sono obiettori di coscienza. Se si stringe la prospettiva di analisi al solo Comune di Verona, l'Azienda Ospedaliera Universitaria Integrata, vede 21 medici dell'ostetricia-ginecologia obiettori su 27. In alcuni casi, per garantire l'espletamento di quello che per legge è un diritto, si è fatto ricorso a consulenze esterne, stipulando per questo convenzioni con altre realtà che, però, sono spesso fuori Provincia e di difficile raggiungimento per l'utenza in particolari condizioni di marginalità.

I dati forniti dall'Istat relativi al 2016, registrano 1.065 Ivg nella Provincia di Verona, di cui 533 vedono protagoniste donne di cittadinanza italiana. Ciò significa che, anche nel territorio veronese, il tasso di abortività delle donne immigrate per l'anno in considerazione è sensibilmente più alto rispetto a quello delle autoctone giacché il $50 \%$ delle Ivg è richiesto da tale categoria di utenti a fronte di una presenza immigrata dell' $11,4 \%$ in Provincia e del 13,9\% nel Comune (Centro Studi e Ricerche Idos, 2018). Tal dato dovrebbe fare riflettere, soprattutto alla luce dei recenti provvedimenti presi dalla politica locale relativamente ai diritti della salute riproduttiva femminile che colpirebbero, così, soprattutto le donne immigrate con minori risorse relazionali. 


\section{Un Giano Bifronte che mangia l'anima}

Per comprendere in che modo abbiamo immaginato di affrontare il tema della paura può essere utile recuperare dall'immaginario mitologico la figura di Giano Bifronte, il dio degli inizi, raffigurato con due volti.

Più esattamente, le paure espresse dalle donne immigrate intervistate costituiscono una metà del dio, mentre l'altra metà è composta dai racconti e dai vissuti delle operatrici sanitarie. A posteriori queste paure ci appariranno come in un gioco di specchi: le une riflesse nelle altre, ma senza per questo venire necessariamente, e costantemente, in contatto.

A ben guardare, però, l'ambivalenza della paura - la sua doppiezza - non si limita alle fila di un ragionamento che abbiamo dovuto annodare per restituire la trama di esperienze vissute, di volta in volta, o dalle donne o dalle operatrici sanitarie, ma consiste, prima di tutto, in quel doppio movimento che ci apprestiamo ad analizzare e che mette la paura al centro di un rapporto dialettico che possiamo esemplificare servendoci di due semplici quesiti: da cosa è prodotta la paura? E cosa, a sua volta, la paura produce?

\subsection{La paura prodotta: le utenti}

Relativamente al primo quesito, vi sono certamente una serie di fattori che contribuiscono a generare il sentimento della paura tra le donne immigrate: a partire dai racconti che esse ci hanno fornito, siamo riusciti a individuare e a distinguere alcuni di questi fattori. Per qualche donna si tratta di un sentimento che può scatenarsi a partire dalla notizia di un parto cesareo, per altre ancora da una gravidanza indesiderata o dall' ammissione di una depressione post partum, o ancora, esso è legato all'incapacità di orientarsi nella frammentazione dei servizi sanitari e alla poca dimestichezza con la lingua del paese di arrivo.

A questi fattori possono sommarsi però ragioni che in molti casi hanno a che vedere con le condizioni materiali e di esistenza in cui versano le donne prive di documenti, di una casa e di un lavoro.

Una donna lungosoggiornante ha raccontato di essersi trovata in estrema difficoltà, nel periodo della gravidanza, a causa delle sue condizioni sociali ed economiche:

Allora io quando sono rimasta incinta ero molto in difficoltà, non avevo nemmeno un posto sicuro. Ho sofferto moltissimo, perché non avevo nemmeno un lavoro [...]. Quando ho smesso di lavorare, poi sono rimasta incinta... quindi mio marito è andato a vivere in un'altra città dove lavora, ma una cosa 
così, come me, una cosa in aria... È stato difficilissimo perché non avevo un posto. Prima dormivo a casa di una mia amica. Dopo, con questo cambiamento, ho avuto paura, dormivo un po' ovunque. Andavo e venivo dalla mia amica perché non si vedeva la pancia, ero magrissima, fino a sette, otto mesi nessuno sapeva che ero incinta. Ai nove mesi si vedeva tantissimo. Quando ero al quinto mese, allora, ho parlato con l'assistente sociale... Sono rimasta un po'... Avevo paura anche per dirglielo, perché lo sapevo come sono difficili [le assistenti sociali] anche perché non avevo lavoro, allora ho pensato che era male... ho pensato che se parlavo non era bene, e allora sono rimasta zitta. Perché con le difficoltà potevano dirmi: “Che hai fatto?". Non lo so, era la mia prima gravidanza e pensavo una cosa scema, perché senza casa, senza lavoro, senza niente... Però succede comunque (Esther, lungosoggiornante).

La paura che affiora dal racconto di Esther suggerisce un'interpretazione che non si limita a incardinare tale sentimento a una dimensione e a un piano squisitamente individuali, come se si trattasse, cioè, del risultato dato dalla sua condizione di senza fissa dimora. Piuttosto, il racconto fornisce degli spunti per provare a ragionare intorno alla dimensione sociale che produce e mette in moto il sentimento della paura della donna; una dimensione rappresentata dalle criticità che emergono nei casi di assistenza alle donne in stato di gravidanza e prive di un posto in cui vivere e che, in ultima istanza, agisce da freno inibitore e impedisce loro di rivolgersi ai servizi incaricati di offrire il necessario supporto, perché convinte che in qualche modo la propria condizione materiale possa mettere inevitabilmente a rischio la possibilità di tenere con sé il proprio figlio una volta nato.

Così, messa di fronte alla circostanza di dover comunicare all'assistente sociale le proprie precarie condizioni materiali, Esther ha preferito la strada del silenzio, almeno fintantoché la "magrezza" glielo ha reso possibile.

Il caso di Esther dimostra come la paura possa definirsi un "pensiero incorporato", generato dall'intreccio tra dimensione individuale, corporea e sociale (Rosaldo, 1984).

La magrezza, così come il rigonfiarsi della pancia, sono aspetti che hanno avuto un ruolo nella sua scelta di nascondere o comunicare il proprio stato; aspetti che a loro volta sono stati presi in considerazione a partire dall'intersezione di elementi attinenti alla sfera individuale della donna - come quello di essere una senza dimora - e alla sfera sociale, come l'opportunità di mettere al mondo un figlio pur trovandosi in una condizione di disagio abitativo e lavorativo.

Le parole di un'altra intervistata mostrano il peso del condizionamento sociale e di figure che incarnano le istituzioni sulle scelte delle donne in ambito di salute riproduttiva: 
Tutto bene, il mio solo problema è il lavoro! Io ho solo questa figlia. Perché non c'è lavoro come si fa a fare figli senza lavoro? Quando tu lavori e hai i soldi puoi fare, anche la mia assistente sociale mi ha detto: "Non si fa così, sola, senza lavoro! Quando trovi qualcuno buono, prima te lo sposi e poi cerchi un lavoro. Lascia tutti i soldi in posta perché devi comprare i vestiti alla bambina, devi mandarla a scuola". Tante cose, anche gli esami in ospedale si pagano! Quando tu sbagli la prima volta, poi ci devi pensare e non devi sbagliare più. Io sono sola. Prima per sette anni mi ha aiutata un italiano. Io gli ho detto che avevo una bambina e avevo tanti problemi. Dopo lui è morto. Quasi un anno fa è morto. All' Arena c'è la mia assistente sociale, lei mi aiuta un po'. L'assistente sociale ti aiuta solo perché ti dà un po' di soldi, però... dopo sei mesi ha detto: “Basta! C'è un'altra persona, non ci sei solo tu!” Però quando tu stai male, sei grave, loro [le assistenti sociali] ti aiutano. Vengono a vedere il bambino per salutarlo... quello sì. Loro non ci danno i soldi. Ci aiutano per la casa, per la scuola dei bambini... perché io non lavoro (Rita, lungosoggiornante).

Anche se il racconto di Rita non riguarda direttamente il sentimento della paura, esso porta allo scoperto le aspettative che ruotano intorno all'idea di maternità e mette in evidenza a quali pressioni le donne siano sottoposte e che tipo di condotte e comportamenti la società si aspetta loro mettano in atto (Giuffrè, 2018; Marabello e Parisi, 2018; Quagliarello, 2018).

Per tornare all'oggetto del nostro contributo, anche in un'altra circostanza da noi osservata, la paura ha messo in moto una spirale di non detti e silenzi. Durante un'intervista con una donna camerunense ospite di un centro di accoglienza in provincia di Verona, ci è stato raccontato che:

Quando loro vanno all'ospedale per fare aborto ci sono altre che hanno vergogna e per quello loro prendono la medicina del loro Paese, questo è molto brutto, rischioso e pericoloso... ma hanno vergogna... Vergogna di andare in ospedale per fare l'aborto [...] e allora loro fanno con la loro medicina del loro Paese, ma questo è molto pericoloso. Secondo me se voi potete aiutare queste ragazze su questo, anche dire che anche se loro sono incinte non è una cosa brutta. Ci sono tanti ragazzi che vendono queste medicine. [...] Adesso ho un'amica che da tre mesi perde sangue perché ha preso questa medicina, ci sono tante signore che vendono questa medicina... La mia amica non vuole dire quello che ha fatto. [...] Se una persona non vuole bambino può andare a parlare con il centro, all'ospedale che è molto bene per far lavare la tua pancia anche fare aborto in sicurezza. Se, invece, prendi la medicina e non sai cosa può fare nella tua pancia per fare aborto, dopo hai problemi che neanche lo sai (Blessed, richiedente asilo). 
Nella conversazione appena riportata, la paura si dispone su due differenti livelli, ognuno dei quali esprime una situazione di "impasse" e aiuta a definire meglio quali siano i rischi che queste donne corrono quando vengono letteralmente "prese" dalla paura.

Il primo livello descrive la condizione di quelle donne che decidono di non portare avanti la gravidanza e che, per paura del giudizio, preferiscono fare ricorso a metodi fai da te, mediante l'assunzione di farmaci dagli effetti abortivi e altamente nocivi (Taliani, 2019). Il secondo livello riguarda, invece, la paura che l'amica di Blessed ha provato al pensiero di dover comunicare a medici e operatori sanitari di aver assunto la "medicina" e di non essersi rivolta a un medico.

Osservando da vicino questi casi, ciò che emerge è che alla base della paura manifestata dalle donne agiscono una o più convinzioni. Nel primo caso, quella di non essere "una buona madre", poiché le condizioni materiali in cui Esther versa non sono adeguate alla nascita e alla crescita di un figlio; nel secondo caso, invece, la convinzione è quella di non essere né "una brava donna", perché la scelta di non portare avanti una gravidanza è, in molte circostanze, ritenuta poco edificante per una donna, né una "brava paziente", dal momento che essa non si è premurata di contattare il medico per interrompere la gravidanza.

Nel quadro di motivazioni che abbiamo elencato, emerge come a produrre la paura contribuiscano anche quei processi e quei meccanismi di stigmatizzazione (dimensione sociale) che, per effetto di categorie e di norme sociali prevalenti, lavora all'individuazione di colpe e responsabilità individuali (piano individuale), invertendo l'ordine di causalità e scambiando gli effetti per le cause. In questo senso, la stigmatizzazione produce la paura tra quegli individui le cui condotte e stili di vita vengono socialmente sanzionati e, non meno importante, essa finisce col generare effetti e ricadute che in qualche caso possono mettere a rischio l'incolumità di chi ne viene colpito (dimensione corporea). La prima di queste ricadute è il silenzio dietro cui si nascondono le persone stigmatizzate, con la conseguenza che, anche in caso di necessità, esse faranno fatica a rivolgersi ai servizi; la seconda riguarda invece il più generale piano d'azione delle politiche sociali e sanitarie, impegnate a destreggiarsi più nelle operazioni di cure che non nelle relazioni di care (Quagliarello, 2016).

\subsection{La paura prodotta: le operatrici}

Le narrazioni delle operatrici che quotidianamente si confrontano con donne immigrate definiscono la metà del volto di Giano che ci consente di indagare e approfondire dimensioni speculari, ma talvolta inedite della paura come prodotto: 
Quest' anno abbiamo avuto una signora del Niger, con una malformazione: è pre-eclamptica e ha vari problemi. Se riuscissimo prima ad avvisare queste persone che devono essere seguite, perché il marito lavora come operaio e lei è da sola qua. Noi dobbiamo fare un sacco di controlli prima di fare l'ecografia. Questo sarebbe un aspetto che ci potrebbe aiutare molto meglio e completerebbe un po' il quadro. [...] Alla fine, invece, quando hanno partorito non le rintracciamo più. [...] Non ritornano, non abbiamo feedback (medico volontario di un ambulatorio per immigrati irregolari).

Un operatore di un centro che offre servizi sanitari, anche in assenza di un regolare permesso di soggiorno, evidenzia come la condizione di irregolarità giuridico-amministrativa generi nelle donne immigrate un sentimento di paura che, in gravidanza, agisce, rendendo faticosi gli accertamenti necessari per valutarne il buon andamento, mentre, nel post partum, spinge le donne a rendersi invisibili e irreperibili.

Alla nascita del figlio, le donne irregolari, anche se in possesso della tessera Stp, si sentono maggiormente esposte a possibili sanzioni o all'espulsione e, per questo, cessano di frequentare il centro, rinunciando, così, a un monitoraggio del proprio stato di salute dopo il parto.

La responsabilità di ciò è, in parte, imputabile al clima politico e sociale che ha caratterizzato il Paese negli ultimi anni.

Il carattere securitario e emergenziale dei provvedimenti normativi riguardanti la disciplina dell'immigrazione (Altin e Sanò, 2017; Basso, 2010; Della Puppa et al., 2020; Gargiulo, 2019) e la retorica costruita a danno degli immigrati hanno generato un timore diffuso che inibisce l'accesso alle cure, esponendo soggetti vulnerabili, come può esserlo una neomadre, a condizioni di marginalizzazione tali da mettere a rischio la propria salute e quella del neonato. La testimonianza dell'operatore del centro non si riferisce ad un caso isolato, ma ad un continuum di situazioni di "invisibilità" nel post partum, come se la nascita del bimbo cancellasse la possibilità di esistenza "visibile" della madre e conseguentemente la possibilità di prendersi cura di sé.

Se la posizione di irregolarità amministrativa spinge le donne a rendersi invisibili, coloro che invece dispongono di un regolare permesso di soggiorno $^{2}$ e si affidano al servizio socio-sanitario pubblico, per trovare aiuto e supporto, manifestano nei suoi confronti, comunque, un sentimento di timore. Le parole di un'assistente sociale di un consultorio privato sono, in tal senso, esplicite:

2. Seppur la stessa intervistata afferma che la transizione da una "categoria" di utenza all'altra da parte delle donne, soprattutto per alcune nazionalità, sia connessa ai loro cicli migratori e biografici, come abbiamo sottolineato nella Nota metodologica. 
La difficoltà che ho avuto è stata con una ragazza nigeriana che era stata vittima di tratta... È molto difficile lavorare con questa utenza, sono molto diffidenti probabilmente per diverse motivazioni e, quindi, non voleva firmare la privacy perché pensava che firmando la privacy magari lei partoriva e c'era una clausola per cui il bimbo lo prendevamo noi e quindi lei non ha firmato la privacy dicendo di volerla farla leggere ad altre persone. Allora gliela ho data da portare a casa e ci siamo riviste in un altro momento... e poi mi ha chiamato un vicino di casa italiano chiedendomi spiegazioni e io gli ho detto tutto. Io dall'inizio spiego tutto sulla documentazione, spiego dove sei e che ti devo fare delle domande per una scheda anagrafica, minimizzo la parte scritta per concentrarci poi a parlare e capire le difficoltà e, infatti, la burocrazia la raccolgo alla fine o all'inizio velocemente. Però c'è la diffidenza perché sono assistente sociale, la paura che si portino via i bambini eccetera. Qua ci salva che siamo un privato, qui le mamme sono più rilassate, è un ambiente informale, le mamme chiacchierano: quello salva un po' di più rispetto a chi lavora in un pubblico e sono anche più sincere, tipo: "Qui c'è scritto che lavoro, ma è un lavoro fittizio perché devo rinnovare il Permesso di soggiorno... ma che rimanga fra me e te" [...] Perché magari dicono: "Non dirlo a quella del servizio sociale - oppure - sono incinta ma ho paura a dirlo a quella del servizio sociale che magari mi porta via il bambino". Lo hanno chiaro, ma perché lo vedono quando vengono qua, perché c'è un po' un'anarchia: non c'è quello sportello che c'è nel pubblico che devi suonare il campanello e dall'altra parte ti risponde... qui la volontaria esce e ti incontra da vicino (assistente sociale di un consultorio del Terzo Settore).

L'intervistata evidenzia che i servizi socio-sanitari pubblici sono vissuti dalle donne come spazi istituzionali e in quanto tali in grado di esercitare un potere bio-medico sui loro corpi e, soprattutto, sulle loro relazioni familiari (Taliani, 2019). Di nuovo, il clima politico, sociale e mediatico fa temere alle donne di essere controllate dall'istituzione e orienta conseguentemente le scelte quotidiane, tra queste, la scelta di confidarsi con un'assistente sociale privata permette da un lato di trovare conforto e supporto e dall'altro di evitare presunti rischi per la propria famiglia. La cura alla relazione agita nel consultorio costituisce la chiave che consente alle operatrici di scardinare quel rapporto asimmetrico tipico delle istituzioni e accompagnare le donne attraverso una relazione di fiducia. La paura del controllo da parte dell'istituzione è, così, stemperata dall'approccio informale che consente l'instaurarsi di legami confidenziali: quando l'operatrice "incontra da vicino" la donna, non solo abbatte un muro invisibile, ma getta le basi per una relazione benefica in termini di salute, non solo per quella donna e famiglia, ma per l'intera collettività.

Il racconto di un'operatrice di un Cas che accoglie richiedenti protezione internazionale testimonia un ulteriore aspetto di come la paura s'incarni nelle quotidianità delle donne immigrate: 
Si aspetta il ciclo che manca. Cioè, a me non è mai successo che uno venga in ufficio a dire: "Guarda ieri ho fatto l'amore senza eh... è successo che... e secondo me... ho paura, cosa facciamo?" Questa cosa ancora non è passata. [...] Forse perché anche temono una sorta di biasimo o di rimprovero da parte nostra..." (operatrice di un Cas).

Il lavoro di operatrici e operatori nel sistema dell'accoglienza è caratterizzato sia dalla rigida adesione alle specificità normative e procedurali che il mandato richiede, sia dal tentativo di costruzione di relazioni di fiducia con le donne accolte. Relazioni caratterizzate, tuttavia, da un' asimmetria strutturale che consente solamente la creazione di rapporti artificiali e obbligati, nonostante i quali operatrici e operatori auspicano di essere a conoscenza, in ogni momento, delle decisioni più intime delle donne accolte, anche quelle che riguardano la vita sentimentale, sessuale e riproduttiva.

L'asimmetria del rapporto comporta inevitabilmente dinamiche di controllo e violenza ai danni delle richiedenti asilo che, di fatto, devono aderire a modelli di comportamento definiti dall' organizzazione "ospitante" (Pasian e Toffanin, 2018) ed è proprio la non adesione a tali modelli a generare il timore di essere rimproverate e/o biasimate.

"Il timore del giudizio altrui è così forte e pervasivo che ci si può vergognare solo immaginando ciò che gli altri potrebbero pensare di noi, anche senza aver compiuto nulla di cui vergognarsi", specifica Turnaturi (2012) e, infatti, oltre al giudizio si teme il rimprovero e il biasimo da parte di chi non solo si occupa del soddisfacimento dei bisogni primari, sanitari, formativi, sociali, ma media anche la possibilità che le donne hanno di ottenere un permesso di soggiorno.

Il ritardo con cui le richiedenti protezione internazionale si rivolgono alle operatrici, talvolta complicando le problematiche rispetto alla cura riproduttiva, sottende, quindi, il timore che l'aver agito in modo non conforme a quanto auspicato dall'organizzazione "ospitante" possa avere delle ripercussioni nell' intero progetto migratorio.

\subsection{La paura produttrice: le utenti}

Se ci limitassimo a considerare la paura come ciò che impedisce l'azione, va da sé che, tra gli effetti che dovremmo tenere maggiormente in conto, vi è senz'altro l'inibizione che questa comporta nell'accesso ai servizi dedicati alla salute. Esso è, infatti, tra quelli che si manifestano con maggiore evidenza e frequenza.

Tuttavia, come avremo modo di mostrare in questo paragrafo, decidere di 
non fare ricorso ai servizi dedicati può, in qualche caso, tramutarsi in una presa di posizione che lascia margini di azione in cui viene messa al centro la soggettività della persona.

Decidere di non fare niente non è necessariamente sinonimo di non-agire, poiché una tale decisione può, in altre parole, indicare che le persone trovano più conveniente agire in altro modo o semplicemente attendere condizioni più favorevoli. Diversamente da quanto abbiamo evidenziato nel precedente paragrafo, la paura che ha motivato le scelte che qui verranno discusse non ha messo in pericolo la vita delle donne e dei nascituri, ma ha assunto piuttosto il ruolo di tattica (De Certeau, 2010).

Una richiedente asilo gambiana, durante l'intervista ha raccontato che senza l'aiuto di un'operatrice dello Sprar in cui ha vissuto durante tutto il periodo della gravidanza non ce l'avrebbe fatta. Grazie al supporto e al lavoro della struttura in cui viveva, Amina ha avuto modo infatti di prendere parte a un corso pre-parto. Tuttavia, giunta quasi alla fine del percorso, la donna ha deciso di interrompere la frequentazione

\begin{abstract}
Arrivata in Italia, per fortuna il parto è andato bene. La mia unica paura era che lei [riferendosi a una delle due gemelle] aveva la testa su, quindi dovevo fare il cesareo. E avevo tanta paura e non ho voluto fare il cesareo è... Ai corsi pre-parto, io capivo bene, loro mi spiegavano bene, ma non ci sono andata più perché avevo paura del cesareo e non volevo sentire che devo fare il cesareo (Amina, richiedente asilo).
\end{abstract}

La paura di dover fare un parto cesareo ha fatto sì che la donna decidesse di interrompere il corso che stava frequentando. In particolare, Amina ha motivato la propria scelta dicendo che "non voleva sentire" di dover fare il cesareo. Messa di fronte all'impossibilità di sottrarsi a un'eventualità che non poteva in alcun modo controllare, ha quindi rinunciato all'ascolto.

In precedenza, abbiamo visto come le donne "prese" dalla paura avessero deciso di non parlare. In questo caso, invece, osserviamo in che modo la non accettazione e il rifiuto di una condizione che si verifica malgrado sé, vengano elaborati e affrontati mediante la scelta di non sentire.

L'esperienza del parto cesareo rientra tra quelle maggiormente affrontate e discusse in letteratura, poiché costituisce un esempio del processo di controllo del corpo femminile attraverso l'uso di pratiche mediche invasive e talvolta non necessarie (Lombardi, 2009, Davis-Floyd, 2001), in questo senso l'Oms ritiene che il tasso ideale di tagli cesari debba essere compreso 
tra il $10 \%$ e il $15 \%^{3}$, mentre la media nazionale è superiore al $33 \%{ }^{4}$. Non sono mancate, nemmeno durante la nostra ricerca, testimonianze di donne straniere che hanno raccontato di aver subito un parto cesareo, seppur, dal loro punto di vista, non sussistevano le motivazioni reali per praticarlo. In altri casi, invece, l'intervento è stato riconosciuto come necessario.

Tra le ragioni che abbiamo ritenuto di dover tenere in considerazione nei casi di parti cesarei praticati su donne straniere, rientra certamente la difficoltà dei medici di dialogare con la donna nei momenti cruciali, laddove è importante che vi sia la massima collaborazione tra i due.

Insieme all'età e al grado di istruzione, l'essere stranieri è uno dei motivi per cui i momenti dedicati all'informazione e al dialogo nella relazione medico-paziente vengono sottovalutati, se non addirittura tralasciati. Molto spesso si ritiene, infatti, che le persone appartenenti a una di quelle categorie non siano effettivamente in grado di capire le informazioni o le istruzioni da seguire e, quindi, dare loro informazioni viene considerato come una perdita di tempo, che sottrae spazio all'operatività (Quagliarello, 2016).

L'etnografia condotta da Chiara Quagliarello (Ibidem) ha mostrato come questa convinzione sia molto spesso il frutto di un pregiudizio del personale sanitario che non sempre trova effettivi riscontri nella realtà, ma che ha, prima di tutto, a vedere con la velocità e i ritmi che si impongono nelle strutture sanitarie e che vanno a detrimento dell'informazione nel suo complesso.

Insieme al fatto che in molte circostanze le persone straniere hanno dimostrato una dimestichezza con la lingua tale da non rendersi necessario nemmeno l'ausilio di una mediazione linguistica, bisogna poi tenere conto - e il caso di Amina sembra suggerire questa interpretazione - di una differenza fondamentale ossia quella tra "non capire" e "non voler sentire".

Nel primo caso sono le complicazioni linguistiche a determinare una delle numerose difficoltà che costellano la relazione medico-paziente; nel secondo caso, invece, il venir meno della relazione è da addebitare a una scelta che proviene direttamente dal soggetto, il quale impone così la sua volontà su quella del sapere medico, dei suoi operatori e dei tecnici. In questo senso, allora, si spiega quanto abbiamo detto in precedenza, laddove abbiamo evidenziato che il non-agire può stare a indicare la volontà del soggetto di mettere al centro se stesso e i propri bisogni, assumendo quindi il ruolo di una tattica.

Una donna lungosoggiornante ci ha raccontato invece della paura che ha

3. https://apps.who.int/iris/bitstream/handle/10665/161442/WHO_ RHR_15.02_ita.pdf; jsessionid=CBAC799C297B5A76410D7EA8C2FB8815? sequence=14.

4. http://www.salute.gov.it/imgs/C_17_pubblicazioni_2881_allegato.pdf. 
provato nel frangente in cui ha compreso di soffrire di depressione post-partum, e dinnanzi alla possibilità di doverlo raccontare al personale sanitario che l'aveva seguita durante il parto. Nelle parole di Maria, l'esperienza del parto aveva rappresentato un incubo, poiché nessuna delle indicazioni che aveva messo nero su bianco sul piano del parto era stata presa in considerazione dai medici che, dopo un lunghissimo travaglio, si erano infine decisi per un parto cesareo. Egualmente, il periodo del post-partum è stato vissuto da Maria in maniera traumatica:

La fine di un incubo e l'inizio di un altro, in realtà. Perché dopo ho avuto la depressione post-parto che l'ho portata avanti per sei mesi. Però avevo tanta paura di andare all'ospedale, di dirlo anche a qualcuno che avevo la depressione e che io, già da sola, mi ero resa conto che avevo la depressione. Stando solo con mio marito nessuno se ne rendeva conto che avevo la depressione. Ero lì, aspettavo e dicevo: quando arriva il viaggio in Perù? Stavo agognando il viaggio in Perù. Pensa che il primo giorno che sono arrivata, ho abbracciato la mia famiglia e ho detto a mio padre: "Ho bisogno di una psicologa". La prima cosa che gli ho detto è stata: "Ho bisogno di una psicologa, per favore!" Avevo paura di dirlo perché non mi fidavo più del sistema italiano. Mi sembrava che erano tutte bugie. Tutte cose che dicono e che poi non si fanno. In uno dei miei ricoveri in ospedale quando ero incinta, ho visto una donna partoriente con la bambina e lei mi aveva detto: "Guarda io con la prima bambina ho avuto la depressione post-parto e ora queste qua non mi lasciano in pace". E io le ho detto: "A che ti riferisci?" E lei: "Da quando ho partorito continuano a dirmi: 'Stai bene? Stai bene? Veramente ti senti bene? E non mi lasciano andare a casa, mi tengono ancora qua"'. Io con tutto quello, più il fatto del parto, della gravidanza che era stato veramente orribile, non riuscivo a dire: "Ho bisogno di aiuto". Io glielo dicevo a mio marito, e lui mi diceva: "Che facciamo?" E io gli dicevo: "No, io non voglio andare". Ero una contraddizione costante (Maria, lungosoggiornante).

Dicevamo di come la paura potesse mettere in moto delle scelte che, sebbene in un primo momento sembrino andare nella direzione di una mancata azione, possono tradursi, invece, in una sospensione e, più esattamente, nell'attesa di condizioni il più favorevoli e confortevoli possibile per il soggetto che le assume. Il lavoro di Pizza e Ravenda (2016) ha messo in evidenza la centralità del tempo nell'analisi della produzione del sapere medico e delle relazioni e dinamiche che esso, a propria volta, produce. Se, relativamente ai parti cesarei, il tempo - concesso o negato - diventa un fattore cruciale per una buona relazione tra medico e paziente, il caso di Maria ci consente di affrontare il tema del tempo, afferrandolo, però, da un punto di vista inedito e, cioè, dalla prospettiva di chi reagisce a una gestione "dall'alto", rallentandolo o accelerandolo a seconda delle esigenze dell'azienda e del 
personale medico, e mettendo in atto la produzione di un altro tempo e, cioè, del proprio tempo. In questi termini, allora, anche la scelta di Maria, provocata dalla paura di dover comunicare il proprio stato di salute a un sistema di cui aveva smesso di fidarsi, può essere interpretata come una tattica, messa in atto dalla donna per sottrarsi ai tempi e a una modalità di gestione della depressione post-partum che, come per il parto, l'avrebbero nuovamente messa nelle condizioni di non essere compresa e ascoltata.

\title{
4.4. La paura produttrice: le operatrici
}

Nel paragrafo precedente, i racconti delle donne intervistate hanno messo in luce come la paura abbia prodotto una non-azione e come questa possa, talvolta, essere interpretata come una tattica. Nelle esperienze delle operatrici emerge un ulteriore aspetto di ciò che la paura può produrre: le conseguenze tragiche di alcune non-azioni. Di seguito, le parole della coordinatrice di un consultorio:

\begin{abstract}
Noi ne abbiamo avute due che hanno perso il bimbo oltre la quarantesima settima, perché non avevano la tessera sanitaria e pensavano di non poter andare in ospedale. Però tu tutta una serie di cose in questi momenti gliele dici, le spieghi che puoi andare al pronto soccorso: "Perdi l'acqua? Vai là! Perdi il sangue? Va là!" Io ce lo ho ancora in mente Jenny, che ha quella bambina disabile... non è solo un tema di visite, tante persone si istruiscono per cui al bisogno sanno che hanno un'emergenza e che in Italia non le mandano via al pronto soccorso, quindi avere i punti dove comunque le accogli serve, soprattutto per la parte informativa, perché quelle due o tre volte che verranno le dici tutta una serie di cose... Però quante hanno paura!? E con quali conseguenze!?" (coordinatrice di consultorio).
\end{abstract}

L'assenza di informazioni adeguate e il timore generato da una posizione di irregolarità amministrativa ha impedito a due donne di recarsi all'ospedale per effettuare i monitoraggi di controllo superata la data presunta del parto, causando la morte dei nascituri.

Ancora una volta emerge come il clima politico e sociale ostile alla popolazione immigrata guidi le donne nelle scelte di cura e non-cura. La disponibilità delle operatrici del consultorio a informare e orientare le donne non è stata sufficiente a scardinare la paura delle ripercussioni amministrative. La scelta di non cercare cure ospedaliere nel tentativo di proteggere sé stesse e il nascituro, magari auspicando in un parto domestico che avrebbe garantito alle donne l'invisibilità, si configura come una scelta soggettiva razionale, 
fondata su un sentimento individuale, plasmato tuttavia da un contesto sociale e politico avverso.

Nei casi, invece, in cui le donne immigrate scelgono di non portare avanti la gravidanza, le operatrici raccontano del timore di queste di non fare in tempo ad accedere all'interruzione volontaria presso una struttura ospedaliera e del ricorso quindi a pratiche abortive fai da te. Nelle parole di un operatore del Network antitratta:

\begin{abstract}
Un elevato numero di Ivg che purtroppo molto spesso sono interruzioni di gravidanza fatte non attraverso i canali ufficiali, ma attraverso l'uso e l'abuso di Cytotec, reperito e pagato sempre tramite le proprie reti, preso a caso... $\mathrm{E}$ questo succede quando non riesci ad avere magari una risposta immediata: se io devo fare la tessera sanitaria, devo prenotare la visita e mi danno l'appuntamento tra due settimane, il test, facciamo fare le beta-Hcg che è lì lì, però non è affidabile, questa qui ha l'ansia... Io posso dirle: "Guarda, in 10 giorni riesco a farti avere l'Ivg in ospedale". Ma se l'amica le dice: "Pigliati cinque pastiglie e risolvi il problema"... Purtroppo, poi, il problema che ci troviamo a gestire è il post aborto (operatore del Network antitratta).
\end{abstract}

Da tale racconto emerge come la paura di non riuscire a interrompere la gravidanza entro il terzo mese, come previsto dalla normativa italiana, guidi le donne nella scelta di procurarsi farmaci abortivi attraverso reti informali. La letteratura evidenzia come, per molte immigrate in condizioni di marginalità, la gravidanza sia un'esperienza non desiderata e il feto sia sovente frutto di una violenza (Quagliarello, 2019). Probabilmente sono queste le ragioni che muovono le donne ad intervenire con rapidità accollandosi il rischio di percorrere strade incerte pur di raggiungere il proprio obiettivo.

La scansione temporale prodotta dall'impianto burocratico e in particolare l'attesa del tempo necessario per lo svolgimento degli esami clinici e per l'individuazione di una data in cui effettuare l'Ivg si sostituisce a quella dei progetti di vita delle richiedenti protezione internazionale e/o vittime di tratta producendo sofferenza psicologica prima, corporea e sociale poi (Pinelli, 2013; Fontanari, 2016).

Il potenziale tempo di attesa dell'intervento, la paura di non riuscire a interrompere la gravidanza e la necessità di tornare velocemente a prostituirsi per saldare il debito contratto per raggiungere l'Italia sono le condizioni che conducono le donne ad effettuare scelte pericolose per la propria salute.

La paura vissuta dalle donne immigrate e testimoniata dai racconti delle operatrici richiama alla memoria la figura di Giano. Le azioni, le non-azioni e le tattiche agite dalle utenti e connesse a differenti aspetti della propria salute riproduttiva si specchiano nelle esperienze delle operatrici chiamate a farsi carico degli esiti nefasti delle azioni mosse da questo sentimento. Una 
tra tutte, la paura delle donne di portare avanti una gravidanza e il ricorso a farmaci con un approccio "fai da te" si riflette nei racconti delle professioniste, che evidenziano come un sistema incapace di offrire risposte celeri e efficaci possa concorrere a produrre paure che sconfinano in situazioni pericolose.

\section{Conclusioni}

Le paure che abbiamo raccontato, incrociando i discorsi delle donne immigrate con quelli delle operatrici, appaiono riflesse le une nelle altre. Esaminate da entrambe le prospettive, esse confermano quanto la dimensione sociale e i profili individuali si intersechino nelle questioni relative alla salute.

Si tratta di due prospettive che sebbene diano luogo a interpretazioni della paura che risentono, in primo luogo, della diversa posizione in cui si ritrovano a esercitare i soggetti in campo, esse sono però egualmente capaci di mostrare la messa in moto e l'incidenza del piano emozionale nelle scelte che indirizzano, ostacolano o, diversamente, modificano i percorsi di salute e di cura delle donne.

Il legame stabilito dagli studiosi tra il nome di Giano Bifronte e la caratteristica del movimento - presente sia nella radice latina ire (andare) sia in quella indoeuropea $y$ - $a a$ (passaggio) - e con esso la doppiezza che domina ogni sua raffigurazione, ci rammentano l'importanza della similitudine, poiché è grazie a questa figura retorica che ci è consentito di rappresentare l'irrappresentabile e, nel caso specifico, di rappresentare quel doppio movimento che è dato dalla paura come prodotto e dalla paura come produttore.

Perché sembra difficile far venire in contatto o in dialogo le due parti lo si intuisce, però, leggendo tra le righe delle testimonianze che abbiamo riportato. Sebbene dai resoconti delle operatrici sia emerso un elevato grado di consapevolezza dei bisogni e delle paure che si impossessano delle donne in gravidanza e nelle fasi successive, ciò che abbiamo riscontrato è che raramente le operatrici e il sistema sanitario, nel suo complesso, valutino l'incidenza degli aspetti sociali nelle questioni di salute. Vale a dire che, molto spesso, si predilige la seconda parte del binomio "socio-sanitario", svalutando quindi l'importanza della prima componente. Ciò si verifica soprattutto all'interno delle aziende ospedaliere, viceversa, nei consultori e nelle associazioni, le relazioni di care tendono a occupare uno spazio relativamente più ampio.

Anche se in questo lavoro non ci siamo concentrati sulle differenze tra ospedali e consultori, alla luce dei racconti riportati, non è difficile scorgere 
l'esistenza di tali differenze e come queste incidano nei percorsi di salute e di cura (Marchetti e Polcri, 2013).

A margine di questi ragionamenti, è però importante sottolineare un altro aspetto, ossia quello relativo alla relazione tra corpo, spazio e tempo. Dall'analisi empirica, questa relazione è apparsa in tutta la sua evidenza e, in maniera particolare, allorquando sia le operatrici sia le utenti hanno raccontato di come la paura sia, in molti casi, il riflesso condizionato di un contrasto, ossia quello tra spazi e tempi delle istituzioni e spazi e tempi delle donne. Un contrasto che mostra in controluce delle relazioni di potere e che, nei fatti, sprigiona il sentimento della paura. Tale sentimento, come abbiamo visto, può però esercitarsi sui corpi e, allo stesso tempo, essere esercitato dai corpi (Rosaldo, 1984), secondo quel meccanismo che regola i rapporti di potere tra i soggetti in campo e che, in ultima istanza, per dirla con De Certeau (2010) esibisce sia le strategie sia le tattiche.

\section{Bibliografia}

Abu-Lughod L. and Lutz C., eds. (1990). Language and the Politics of Emotion. Cambridge: Cambridge University Press.

Altin R. e Sanò G. (2017). Richiedenti asilo e sapere antropologico. Una introduzione. Antropologia Pubblica, 3, 1: 7-34; doi: 10.1473/anpub.v3i1.94.

Ambrosini M. (2014). Parenting from a Distance and Processes of Family Reunification: A Research on the Italian Case. Ethnicities, 15, 3: 440-459; doi: 10.1177/ 1468796814547059.

Baldassar L. (2010). Ce sentiment de culbabilitè: Réflexion sur la relation entre émotions et motivation dans les migrations et le soin transnational. Journal Recherches sociologiques et anthropologiques, 41, 1: 15-37; doi: 10.4000/rsa.185.

Basso P., a cura di (2010). Razzismo di stato. Stati Uniti, Europa, Italia. Milano: FrancoAngeli.

Bolton S.C. (2005). Emotions Management in the Workplace. Basingstoke: Palgrave.

Bonfanti S. (2012). Farsi madri. L'accompagnamento alla nascita in una prospettiva interculturale. Torino: Cirsde.

Bordonaro L. (2005). L'amor come stile culturale. Auto-poiesi e strategie emozionali tra i giovani di Bubaque, Guinea Bissau. Antropologia, 5, 6: 127-149; doi: 10.14672/ada2005135\%25p.

Bourdieu P. (1972). Esquisse d'une théorie de la pratique. Genève: Droz.

Bourdieu P. (1998). La Domination masculine. Paris: Éditions du Seuil.

Brooks A. and Simpson R. (2013). Emotions in Transmigration: Transformation, Movement and Identity. London: Palgrave Macmillan.

Bryceson D. and Vuorela U. (2002). The Transnational Family: New European Frontiers and Global Networks. Oxford: Berg. 
Bustamante J.J. and Alemàn C. (2007). Perpetuating Split-Household Families. The Case of Mexican Sojourners in Mid-Michigan and their Transnational Fatherhood Practices. Migraciones Internacionales, 4, 1: 65-86.

Carling J. (2008). The Human Dynamics of Migrant Transnationalism. Ethnic and Racial Studies, 31, 8: 1452-1477; doi: 10.1080/01419870701719097.

Cattaneo M.L. e Dal Verme S. (2005). Donne e madri in migrazione. Prospettive transculturali e di genere. Milano: Unicopli.

Censis (2015). I nuovi pellegrini. Roma: Fondazione Farmafactoring e SanArtLab.

Centro Studi e Ricerche Idos. (2018). Dossier Statistico Immigrazione 2018. Roma: Idos.

Cerulo M. (2018). Sociologia delle emozioni. Autori, teorie, concetti. Bologna: il Mulino.

Cerulo M. (2019). The Sociological Study of Emotions: Interactionist Analysis Lines. Italian Sociological Review, 9, 2: 183-194; doi: 10.13136/isr.v9i2.275.

Charsley K. (2005). Unhappy Husbands. Masculinity and Migration in Transnational Pakistani Marriages. Journal of the Royal Anthropological Institute, 11, 1: 85-105; doi: 10.1111/j.1467-9655.2005.00227.x.

Charsley K. and Shaw A. (2006). Rishtas: Adding Emotion to Strategy in Understanding British Pakistani Transnational Marriages. Global Networks, 6, 4: 405421; doi: 10.1111/j.1471-0374.2006.00152.x.

Crocetti E., Manneschi G. and Visioli C.B. (2010). Risk of invasive cervical cancer and cervical intraepithelial neoplasia grade III in central Italy by area of birth. Journal of Medical Screening, 17: 87-90; doi: 10.1258/jms.2010.009100.

Davis-Floyd R. (2001). The technocratic, humanistic and holistic paradigms of childbirth. International Journal of Gynecology and Obstetrics, 75: 5-23.

De Certeau M. (2010). L'invenzione del quotidiano. Edizioni Lavoro: Roma.

Della Puppa F. (2018). Ambivalences of the emotional logics of migration and family reunification: Emotions, experiences and aspirations of Bangladeshi husbands and wives in Italy. Identities, 25, 3: 358-375; doi: 10.1080/1070289X.2016. 1238381.

Della Puppa F., Gargiulo E. e Semprebon M. (2020). Per una critica delle politiche migratorie italiane: fare ricerca, prendere posizione. In: Martoriano N. e Prearo M., a cura di, Politiche della vulnerabilità. Contesti di accoglienza e migranti Lgbt. Pisa: Ets.

Ferrero M. (2010). Il 'pacchetto sicurezza': dall'integrazione subalterna degli immigrati, alla loro criminalizzazione. In: Basso P., a cura di, cit.: 423-437.

Fields J., Copp M. and Kleinman S. (2007). Symbolic Interactionism, Inequality, and Emotions. In: Stets J.E. and Turner J.H., eds., Handbook of the Sociology of Emotions (pp. 155-178). New York: Springer.

Fontanari E. (2016). Soggettività en transit. (Im)mobilità dei rifugiati in Europa tra sistemi di controllo e pratiche quotidiane di attraversamento dei confini. Mondi Migranti, 28, 1: 39-60; doi: 10.3280/MM2016-001003.

Gargiulo E. (2018). Una filosofia della sicurezza e dell'ordine. Il governo dell'immigrazione secondo Marco Minniti. Meridiana, 91: 151-173.

Geertz C. (1973). The Interpretations of Cultures. New York: Basic Books. 
Giuffrè M. (2018). Essere madri d'oltreoceano: maternità transnazionale delle donne capoverdiane in Italia”. In: Giuffrè M., a cura di, Essere madri oggi tra biologia e cultura. Etnografie della maternità nell'Italia contemporanea. Pisa: Pacini.

Harré R., ed. (1986). The Social Construction of Emotions. Oxford: Blackwell.

Harris S. (2015). An Invitation to the Sociology of Emotions. London-New York: Routledge.

Hochschild A.R. (1979). Emotion Work, Feeling Rules and Social Structure. American Journal of Sociology, 85, 3: 551-575.

Ketler S. (2000). Preparing for motherhood. Medical Anthropology Quarterly, 14, 2: 138-158; doi: 10.1525/maq.2000.14.2.138.

Lindquist A.J. (2009). The Anxieties of Mobility. Migration and Tourism in the Indonesian Borderlands. Hawai: University of Hawaii Press.

Lombardi L. (2009). La medicalizzazione della riproduzione umana: il corpo e il genere. Salute e Società, 8, 2: 172-188; doi: 10.3280/SES2009-002012.

Lombardi L. (2005). Società, culture e differenze di genere. Percorsi migratori e stati di salute. Milano: FrancoAngeli.

Lutz C. and White M.G. (1986). The Anthropology of Emotions. Annual Review of Anthropology, 15: 405-436.

Mangone E. e Masullo G. (2010). Tra integrazione e particolarismi: gli immigrati e il ricorso ai servizi sanitari. In: Ammaturo N., De Filippo E. e Strozza S., a cura di, La vita degli immigrati a Napoli e nei paesi vesuviani. Milano: FrancoAngeli.

Marabello S. e Parisi M.L. (2018). "Diventare madri e Hiv. Saperi, senso del tempo e soglie corporee". In: Giuffrè, M., a cura di, Essere madri oggi tra biologia e cultura. Etnografie della maternità nell'Italia contemporanea. Pisa: Pacini.

Marchetti M. e Polcri C. (2013). Gravidanza, parto, puerperio in un contesto d'immigrazione: un approccio antropologico per la calibrazione culturale dei servizi socio-sanitari. AM Rivista della Società italiana di antropologia medica, 35-36: 247-278.

McCarthy E.D. (2017). Emotional Lives. Dramas of Identity in an Age of Mass Media. Cambridge: Cambridge University Press.

Ministero della Salute (2017). Relazione del Ministro della Salute sulla attuazione della legge contenente norme per la tutela sociale della maternità e per l'interruzione volontaria di gravidanza. Dati definitivi 2016. Roma: Ministero della salute.

Parreñas R.S. (2008). Transnational Fathering: Gendered Conflicts, Distant Disciplining and Emotional Gaps. Journal of Ethnic and Migration Studies, 34, 7: 1057-1072; doi: 10.1080/13691830802230356.

Pasian P. e Toffanin A.M. (2018). Richiedenti asilo e rifugiate nello Sprar. Contraddizioni nel sistema d'accoglienza. Mondi Migranti, 34, 1: 127-145; doi: 10.3280/MM2018-001007.

Pasini N. e Pullini A. (2002). Nascere da stranieri. I punti nascita in Lombardia. Milano: Fondazione Ismu.

Pinelli B. (2013). Migrare verso l'Italia. Violenza, discorsi, soggettività. Antropologia, 15: 7-20; doi: 10.14672/ada2013182\%25p. 
Pizza G. e Ravenda A. (2016). Esperienza dell'attesa e retoriche del tempo. L'impegno dell' antropologia nel campo sanitario. Introduzione. Antropologia Pubblica, 2, 1: 29-44; doi: 10.1473/anpub.v2i1.17.

Pussetti C. (2005). Introduzione. Discorsi sull'emozione. Antropologia, 5, 6: 5-14; doi: $10.14672 /$ ada2005129\%25p.

Quagliarello C. (2019). Salute riproduttiva, genere e migrazioni. Il continuum di violenze nei vissuti di donne e madri "dalla pelle nera". Mondi Migranti, 37, 1: 195-216; doi: 10.3280/MM2019-001011.

Quagliarello C. (2018). Rappresentazioni e pratiche dell'esperienza di maternità tra le donne senegalesi dell'Alta Val d'Elsa. In: Giuffre, M., a cura di, Essere madri oggi tra biologia e cultura. Etnografie della maternità nell'Italia contemporanea. Pisa: Pacini.

Quagliarello C. (2016). Tempo concesso e tempo negato nella relazione medico-paziente. Un'applicazione del sapere antropologico alla pratica del consenso informato. Antropologia Pubblica, 2, 1: 117-134; doi: 10.1473/anpub.v2i1.23.

Quaranta I. e Ricca A. (2012). Malati fuori luogo. Milano: Cortina.

Reddy W.M. (1999). Emotional liberty: politics and history in the anthropology of emotions. Cultural Anthropology, 14, 2: 256-288; doi: 10.1525/can.1999.14.2. 256.

Rosaldo M. (1984). Toward an Anthropology of Self and Feeling. In: Le Vine A.R. and Shweder A.R., eds., Culture Theory. Essays on Mind, Self, and Emotion (pp. 135-157). Cambridge: Cambridge University Press.

Rossi P. e Tognetti M. (2017). Oltre la traduzione: il mediatore linguistico-culturale come (s)oggetto di confine. In: Miele F. e Piras E.M., a cura di, Mettere la cura in pratica. Professioni e scene della cura fuori dagli ospedali (pp. 73-98). Milano: Ledizioni.

Sandstrom K.L., Lively K.J., Martin D.D. and Fine G.A. (2013). Symbols, Selves, and Social Reality: A Symbolic Interactionist Approach to Social Psychology and Sociology. Oxford: Oxford University Press.

Skrbiš Z. (2008). Transnational Families: Theorising Migration, Emotions and Belonging. Journal of Intercultural Studies, 29, 3: 231-246; doi: 10.1080/ 07256860802169188 .

Spadea T., Zengarini N. and Kunst A. (2010). Cancer risk in relationship to different indicators of adult socioeconomic position in Turin, Italy. Cancer Causes Control, 21, 7: 1117-1130; doi: 10.1007/s10552-010-9539-0.

Taliani S. (2019). Il tempo della disobbedienza Per un'antropologia della parentela nella migrazione. Verona: Ombre Corte.

Tarabusi F. (2014). Politiche dell'accoglienza, pratiche della differenza. Servizi e migrazioni sotto la lente delle politiche pubbliche. Archivio Antropologico Mediterraneo, 16, 1: 45-62.

Terraneo M. e Tognetti Bordogna M. (2018). È possibile studiare la relazione tra immigrazione e salute in Italia? Molte questioni, ancora pochi dati. Rassegna Italiana di Sociologia, 4: 789-814; doi: 10.1423/92204.

Thoits P. (1996). Managing the Emotions of Others. Symbolic Interaction, 19: 85109; doi: 10.1525/si.1996.19.2.85. 
Toffanin A.M. (2015). Controcanto. Donne latinoamericane tra violenza e riconoscimento. Milano: Guerini.

Tognetti Bordogna M. (2012). Accesso ai servizi sanitari e costruzione della cittadinanza dei migranti. Autonomie locali e Servizi Sociali, 1: 111-124; doi: $10.1447 / 37770$.

Tognetti Bordogna M., a cura di (2008). Disuguaglianze di salute e immigrazione. Milano: FrancoAngeli.

Turnaturi G. (2012). Vergogna. Metamorfosi di un'emozione. Milano: Feltrinelli.

Turner J.H. and Stets J.E. (2005). The Sociology of Emotions. Cambridge: Cambridge University Press.

van Zomeren M. (2016). From Self to Social Relationships. Cambridge: Cambridge University Press 